\title{
Boredom proneness is associated with noisy decision-making, not risk-taking
}

\author{
Ofir Yakobi ${ }^{1}\left[\right.$. James Danckert ${ }^{1}$
}

Received: 18 October 2020 / Accepted: 22 March 2021 / Published online: 8 April 2021

(c) The Author(s), under exclusive licence to Springer-Verlag GmbH Germany, part of Springer Nature 2021

\begin{abstract}
Previous research shows that individuals who tend to get bored frequently and intensely—-the highly boredom prone-are more likely to engage in risky behaviors. However, these studies are based largely on self-reports. Here we address this gap and suggest that noisy decision-making (DM) is a potential driver for this relationship between boredom proneness and risktaking. In Study 1, eighty-six participants completed the Balloon Analogue Risk Task (BART) while EEG was recorded. We found blunted feedback processing with higher boredom proneness, as indexed by reduced feedback-P3 amplitudes. Risk taking, as indexed by the BART, was not higher in the highly boredom prone. In Study 2a $(N=404)$ we directly tested the noisy DM hypothesis in an online sample using a binary choice task, and found that with higher boredom proneness, participants were more likely to alternate between choices on a trial-to-trial basis, but were not more likely to choose the risky alternative. These findings were replicated in a new sample (Study $2 b$ ), and extended to the Iowa Gambling Task (IGT; Study 3). In the IGT we found increased choice switching and reduced feedback sensitivity with higher boredom proneness. Once again, higher risk taking as indexed by the IGT was not evident in the highly boredom prone. Overall, our findings suggest that boredom proneness is associated with noisy decision-making (i.e., a tendency to alternate more between choice options regardless of risk level), and not risk-seeking per se. That is, the highly boredom prone are not necessarily attracted to risks, but rather, may be insensitive to risks due to reduced feedback sensitivity.
\end{abstract}

Keywords Boredom $\cdot$ Risk taking $\cdot$ Decision making $\cdot$ Feedback P3 $\cdot$ Feedback related negativity

\section{Introduction}

It is widely documented that boredom - or more specifically the individual trait of boredom proneness (Eastwood et al. 2012), is associated with risky behaviors. Individuals high on trait boredom proneness (i.e., the tendency to experience boredom more intensely and frequently; Tam et al. 2021) are more likely to binge drink and use drugs (Biolcati et al. 2018; Iso-Ahola et al. 1991), use a mobile device while driving (Oxtoby et al. 2019) and report pathological gambling (Blaszczynski et al. 1990). These findings, however, are based solely on self-reports and are focused on boredom proneness, neglecting any potential association

Communicated by Melvyn A. Goodale.

Ofir Yakobi

ofiryakobi@gmail.com

1 Department of Psychology, University of Waterloo, Waterloo, ON, Canada with in-the-moment feelings of boredom (i.e., state boredom, described as the aversive feeling of failing to occupy one's mind with a satisfying activity; Eastwood et al. 2012).

People experience boredom when they want to be engaged with a task in some satisfying or meaningful manner, but are unable to do so (Danckert 2019; Danckert et al. 2018; Eastwood et al. 2012). Thus, boredom rises when the environment presents us with something better to do than what we are currently engaged in. This notion was formalized in the "opportunity cost" hypothesis of boredom (Kurzban et al. 2013): boredom as a self-regulatory signal, indicating how well we are currently maximizing our mental resources to achieve our goals. This notion was empirically put to the test when participants were asked to sit patiently in a room, with nothing but their thought (Struk et al. 2020). When the room was filled with potentially engaging activities (e.g., laptop, a half-finished puzzle) that participants were told not to engage with, their reported levels of state boredom were higher, compared with participants who waited in an empty room. The opportunity cost hypothesis captures the notion 
that boredom is not sensitive to the current activity (or lack of activity) per se, but rather to the range of possible actions available to the individual.

Trait boredom proneness is associated with negative consequences in the cognitive, affective and behavioral domains. Boredom proneness is associated with increased symptomology of attention deficit-hyperactivity disorder (ADHD; Malkovsky et al. 2012), executive dysfunction, hyperactivity and inattention (Gerritsen et al. 2014), as well as with increased aggression (particularly hostility) and depression (Isacescu et al. 2017). Highly boredom prone individuals are at risk for problematic gambling, substance abuse, impulsivity and risk-taking (Biolcati et al. 2018; Blaszczynski et al. 1990; Dahlen et al. 2005).

When it comes to the relationship between boredom and actual behavior (e.g., risky decisions in decision-making tasks), in contrast to self-reports, the literature is rather scarce. In one study, Campbell (2013) measured trait boredom as boredom susceptibility, a subscale of the sensation seeking scale (Zuckerman et al. 1978), and risk-taking, using the Balloon Analogue Risk Task (Lejuez et al. 2002). Surprisingly, they found a negative relationship between boredom susceptibility and risk-taking. However, as the authors noted, this may have been due to the fact that risk-taking in this task is confounded with time-on-task, (i.e., participants who choose to take more risks, have to spend more time on the task). Similarly, we found only two studies that have examined state boredom and behavior directly (K1lıç et al. 2020; Miao et al. 2019). In two experiments, Kılıç and colleagues (2020; Studies 2, 3) found state boredom to be positively correlated with risky decision making. In their study 2 , participants made 24 choices between a safe option (certain gain) and a risky option (50\% chance to win double the certain gain from the safe option), and in study 3 they made 64 decisions between safe and risky options that both entailed risk (i.e., there was no certain outcome). Results showed that with higher levels of state boredom, participants were more likely to make risky decisions. Similarly, in another study, two experiments investigated the causal effect of induced state boredom on risk-taking behavior and reported similar findings: when induced to feel boredom, participants showed a preference for the risky prospects (Miao et al. 2019; Studies 2, 4). Another study, however, failed to show an effect of a boredom mood induction on risky decision making in a sample of ADHD patients (Matthies et al. 2012).

What drives highly boredom prone individuals to report that they are more likely to engage in risky behaviors across multiple domains (Kılıç et al. 2020, Study 1), for instance, to break social distancing rules during the COVID-19 pandemic (Boylan et al. 2020)? A few mechanisms have been proposed to account for the purported relationship between boredom proneness and risk-taking. Generally, risky decision making can be seen as a maladaptive response to aversive affect (Blaszczynski et al. 1990), including boredom. In that context, risky behavior may have a functional role in alleviating in-the-moment feelings of boredom which the highly boredom prone experience more intensely (Tam et al. 2021). For example, boredom prone adolescents may binge drink because they expect alcohol to enhance disinhibition and socialization (Biolcati et al. 2016). Similarly, Miao et al. (2019) found that the motivation to seek stimulation mediated the relationship between state boredom and risk-taking. Indeed, those high on boredom proneness are also high on self-reported impulsivity and sensation seeking (Dahlen et al. 2004; Dahlen et al. 2005; Kass Vodanovich 1990). Moreover, the highly boredom prone have been shown to have diminished ability to self-regulate (Mugon et al. 2018; Struk et al. 2016; Wolff et al. 2020) and difficulty with self-control (Isacescu 2018; Isacescu et al. 2017; Wolff et al. 2020). Thus, there are several potential routes by which boredom, and particularly boredom proneness, could influence decision making and lead to a higher prevalence of risk-taking behaviors.

Another potential contributor to the relation between boredom and risk-taking is the dysregulation of attention, often seen with state and trait boredom. Highly boredom prone individuals are more likely to exhibit symptoms of ADHD and to report lapses in everyday attention (Carriere et al. 2008; Malkovsky et al. 2012). In lab settings, participants often exhibit poorer performance in attention tasks when they experience in-the-moment feeling of boredom (Danckert Merrifield 2018; Eastwood et al. 2012; Hunter Eastwood 2018; Yakobi et al. 2021). For example, a recent study exploring the relationship between boredom and attention dysregulation in children found that those diagnosed with ADHD reported higher levels of trait boredom proneness, and after completing the Conners Continuous Performance Task (CPT) - higher levels of state boredom (Hsu et al. 2020). In addition, their reported state boredom was correlated with inattention during the CPT.

In sustained attention tasks, boredom and its associated inattention may be expressed in poorer performance. Similarly, boredom has negative consequences in decisionmaking tasks, expressed as reduced attention to the structure of the task and diminished sensitivity to feedback (Kılıç et al. 2020). Hence, feedback processing may be hindered in highly boredom prone individuals, an effect that could potentially be exacerbated by in-the-moment feelings of boredom - leading to suboptimal decision-making. One potential approach to evaluating the effects of boredom on feedback processing, is the use of neurophysiological markers. The feedback-related negativity event-related potential (FRN; Gehring Willoughby 2002; Miltner et al. 1997) is a frontocentral waveform elicited in response to task-relevant feedback, approximately $250 \mathrm{~ms}$ after feedback onset. 
A related component is the feedback-P3 (Martín 2012), a parietally distributed positive deflection, peaking approximately $300 \mathrm{~ms}$ after feedback onset. These two event-related potentials (ERPs) are recorded using scalp EEG, and their amplitude is known to be modulated by feedback. These ERP changes can then be correlated with both state and trait variables to account for individual differences in feedback processing. In one related study, adolescents displaying problematic internet use, a behavior often correlated with boredom proneness (Elhai et al. 2018; Skues 2016), were shown to have reduced FRN and feedback-P3 (Yau et al. 2015). The direct relationship between state and trait boredom, and feedback processing, however, is yet to be determined.

In the present work we aimed to bridge the gap between these two main themes depicted in the boredom literature: boredom and inattention, and boredom and risk-taking. We suggest that these two phenomena are related, and potentially reflect a common mechanism of deficient regulation of attention. Furthermore, we intended to shed light on the relatively underexplored relation between trait boredom proneness and risk-taking in the lab.

We present four experiments that complement each other in the information they provide. Study 1 is an EEG investigation allowing for direct examination of risky decision making and the neurophysiological markers of feedback processing in a well-validated decision-making task. Studies $2 \mathrm{a}$ and $2 \mathrm{~b}$ used a binary, feedback-based decision-making task designed to observe risky decision making and choice switching. In study 3, we chose the Iowa Gambling Task (Bechara, Damasio, Damasio, Anderson, 1994) which has been extensively used for differentiating risky decision making in healthy and clinical participants using a computational modeling approach.

\section{Study 1-risky decision making and feedback processing in the BART}

In this study we used the well-validated Balloon Analogue Risk Task (BART; Lejuez et al. 2002), while recording participants' electrical brain activity. We investigated risky decision making in this task and the relationship between state boredom, boredom proneness and feedback-locked ERPs.

The BART was shown to have high criterion validity as it correlates with a range of risky decision-making behaviors including increased alcohol consumption, smoking, and other risky behaviors (Fernie et al. 2010; Hanson et al. 2014; MacPherson et al. 2010). We hypothesized that (1) state boredom and boredom proneness would be positively correlated with risky decision making (see below for the quantitative metric of this), and (2) state boredom and boredom proneness would be negatively correlated with the magnitude of the FRN and feedback-P3 ERPs. We decided not to manipulate state boredom, because in ours and others' experience (unpublished data from our lab, but also see Hunter Eastwood, 2018's Table 4, p. 2488), boredom mood inductions tend to be short-lived with ratings of state boredom increasing as participants complete the tasks intended to measure the consequence of the mood induction. That is, our tasks perform better as boredom mood inductions than any direct mood induction. This is problematic as any control group (i.e., a group who underwent no boredom mood induction or an interest mood induction) rapidly reaches the same level of boredom during task performance as does the treatment group. In addition, mood inductions necessitate between subjects designs which in turn demand much larger sample sizes. For those reasons, we chose to focus on spontaneous levels of self-reported state boredom as experienced by the participants after completing our tasks.

\section{Participants}

Eighty-six undergraduate students (64 females) aged from 18 to 23 years $(M=19.8, \mathrm{SD}=1.44)$ participated in the study. They completed questionnaires as part of an online survey administered to a larger sample of undergraduate students at the University of Waterloo and took part in the study for course credits and a monetary reward (detailed below). All participants gave written informed consent prior to participating and the protocol received approval from the University of Waterloo's Office of Research Ethics. Participants were healthy with normal or corrected-to-normal vision.

\section{Materials}

\section{Balloon analogue risk task (BART)}

We deployed a variant of the BART (Lejuez et al. 2002) in which participants face a series of 100 balloons one at a time, with each one preset to explode at a random ${ }^{1}$ point (drawn from a 1-128 uniform distribution). Participants choose how many times they would like to 'pump' the balloon using a slider (Fig. 1). Once the number of pumps was chosen, they pressed a button labeled "pump" and received feedback. If the balloon popped (i.e., they chose a pump number beyond the popping point) they would not receive any points for that trial. If the number of pumps chosen did not breach the popping point, they gained a point for each pump (e.g., if the popping point was 78 and the participant chose 42 pumps, they gained 42 points). Feedback appeared

\footnotetext{
${ }^{1}$ The sequence of popping points was generated once, with this same sequence then used for all participants, to reduce variability.
} 


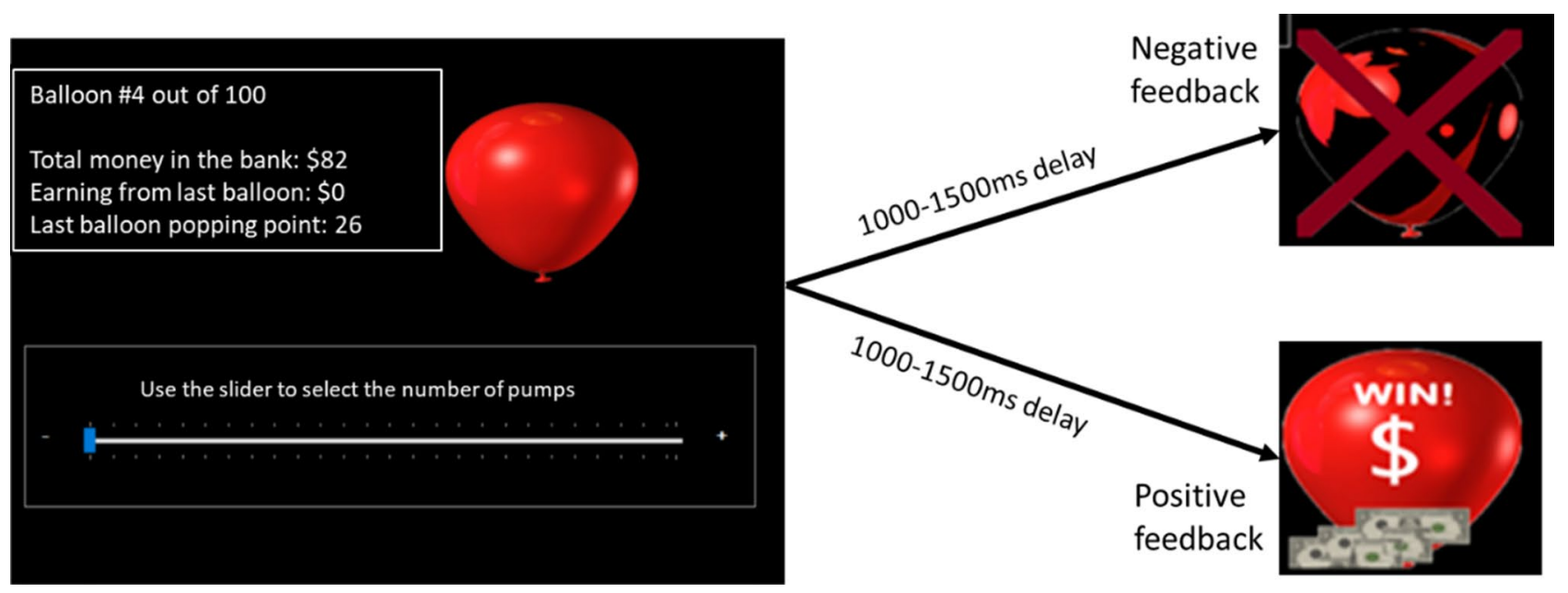

Fig. 1 The Balloon Analogue Risk Task used Study 1. Left screen shows the main task, with the possible feedback screens shown on the right

after $1000 \mathrm{~ms}$ (with an additional 0-500 ms random delay to account for expectancy effects) for a fixed duration, unrelated to the number of pumps. If the balloon popped, the popping point was provided to the participant (Fig. 1). This variation, unlike the classic BART, does not confound risktaking with time on task, which could complicate any interpretation of the relationship between state boredom and risktaking (e.g., Campbell et al. 2013). In addition, it is more suited for recording ERPs (Pleskac et al. 2008).

\section{Short boredom proneness scale (sBPS)}

Trait boredom proneness was measured using eight items rated on a 1 (strongly disagree) to 7 (strongly agree) Likert scale (Struk et al. 2017). Example items include "I find it hard to entertain myself," and "Much of the time, I just sit around doing nothing." Items were then averaged for a composite trait boredom proneness score. This scale showed high internal consistency in our sample (Cronbach's $\alpha=0.89$ ).

\section{State boredom probes}

Participants rated how bored they were on a 1 (not at all) to 9 (highly) scale, using a slider. There were two boredom probes, one collected just prior to task commencement (but after EEG setup had been completed) and one after completion of the task (and before removing the EEG setup from participants).

\section{EEG recording}

Electrophysiological data were recorded using Biosemi Active-Two amplifier with active $\mathrm{Ag} / \mathrm{AgCl}$ electrodes on 32 scalp sites (10-20 system). Additional electrodes were placed over the left and right mastoids as linked reference, next to each outer canthus for horizontal ocular movements, and one below the right eye for detecting vertical ocular movements. Data were sampled at $2048 \mathrm{kHz}$ and down-sampled offline to $256 \mathrm{~Hz}$. The complete preprocessing pipeline and data extraction procedure are detailed in Appen$\operatorname{dix} \mathrm{A}$. All of the analyses were conducted using EEGLAB (Delorme Makeig 2004) and ERPLAB (Lopez-Calderon Luck 2014).

\section{Event-related potentials (ERPs)}

We extracted feedback-locked ERPs and analyzed the difference wave by subtracting win feedback activity from loss feedback activity. For each participant the ERPs were quantified as the negative peak amplitude of the difference wave between 220-320 ms after feedback onset (feedback-related negativity; FRN), and the positive peak found 350-450 ms after feedback onset (feedback-P3), at the mid-central electrode, $\mathrm{Cz}$. The corresponding latencies were calculated as the peak latency within these time windows, for each ERP.

\section{Procedure}

Participants were seated $50 \mathrm{~cm}$ from a 17 " computer screen and were attached with EEG electrodes. Resting state EEG was recorded with eyes closed and eyes open for a period of two minutes each, followed by two tasks performed in a random order: The BART and a go/no-go task (not reported here). The BART started with a state boredom probe, written and visual instructions, followed by four practice trials. Upon completion of the task, a second state boredom probe was administered. Participants were paid between $\$ 1.5$ and $\$ 3.5$ (CAD) contingent on the number of points they gained in the task using a 1 cent per 10 points conversion rate. 
Table 1 Correlation matrix and descriptive of the behavioral and self-report variables in the study

\begin{tabular}{lllllllll}
\hline & 1 & 2 & 3 & 4 & 5 & 6 \\
\hline 2 & Boredom post-task & 0.126 & $4.06(2.09)$ & & & & \\
3 & Boredom pre-task & 0.125 & $0.465^{* * *}$ & $3.99(2.04)$ & & & \\
4 & Pumps & 0.121 & -0.085 & -0.003 & $49(13.9)$ & & & \\
5 & Decision time & 0.114 & $-0.256^{*}$ & -0.142 & 0.028 & $4.36(1.43)$ & $-3.17(5.5)$ \\
6 & FRN amplitude & -0.111 & 0.067 & 0.115 & -0.051 & -0.108 & $0.418^{* * *}$ \\
7 & fP3 amplitude & $-0.22^{*}$ & -0.098 & 0.081 & $-0.269^{*}$ & -0.08 & $7.13(7.25)$ \\
\hline
\end{tabular}

Values on the diagonal represent mean (standard deviation)

$* p<0.05, * * p<0.01, * * * p<0.001$
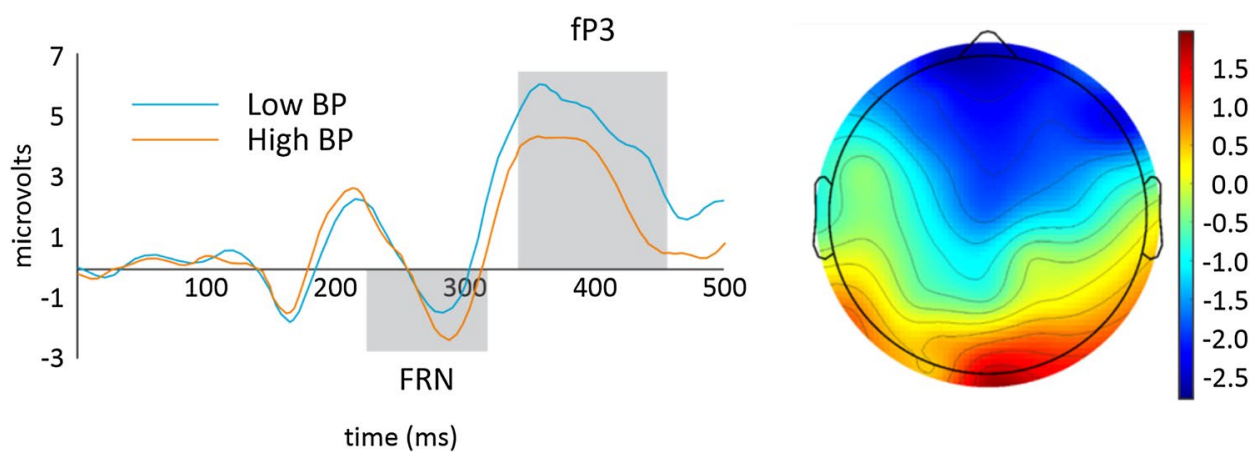

Fig. 2 (Left) The grand average feedback-locked difference wave for boredom proneness (median-split). Gray rectangles represent the FRN and feedback-P3 time windows. Higher absolute values represent better discrimination between positive and negative feedback

\section{Results}

The correlations, means and standard deviations of the behavioral and self-reported variables are described in Table 1.

\section{Boredom and behavior}

To test whether boredom was associated with risky decision making, we calculated the correlation between boredom proneness, state boredom, and the number of pumps made in the BART. The correlation between boredom proneness and risky decision making was not significant $(r=0.121$, $p=0.269$ ). Pre- and post-task boredom ratings were positively correlated $(r=0.464, p<0.001)$, but neither ratings were significantly correlated with risk-taking $\left(r_{\text {pre }}=0.125\right.$, $\left.p=0.251 ; r_{\text {post }}=0.126, p=0.246\right)$. Next, we correlated decision-time - the time from trial start to pressing "pump", with the state and trait boredom metrics. There was a significant correlation between decision-time and post-task boredom $(r=-0.256, p=0.018)$, but not with pre-task boredom trials. (Right) Topographic map of the corresponding difference between high and low boredom prone individuals at $360 \mathrm{~ms}$ after feedback onset

$(r=-0.142, p=0.192)$ or boredom proneness $(r=0.114$, $p=0.298)$. These findings suggest that as the task wore on, feelings of state boredom increased leading to an increase in impulsivity (i.e., faster response times), in the absence of any relation with risky behavior per se.

\section{Boredom and ERPs}

Due to a technical error, EEG recordings from three participants were lost. For the remaining 83 participants, we did not find significant correlations between the FRN and pre-task boredom $(r=0.115, p=0.3)$, post-task boredom $(r=0.067$, $p=0.55)$ or boredom proneness $(r=-0.111, p=0.318)$. There was a negative correlation between the feedbackP3 and boredom proneness ( $r=-0.22, p=0.046$; Fig. 2), but not with pre- or post-task state boredom $\left(r_{\text {pre }}=0.08\right.$, $\left.p=0.466 ; r_{\text {post }}=-0.098, p=0.379\right)$. There were no significant correlations between state boredom or boredom proneness and latency. 


\section{Summary and discussion}

Surprisingly, we did not find evidence for increased risky decision making with state boredom or boredom proneness in the decision-making task used here. Our findings did show that in-the-moment feelings of boredom were correlated with impulsivity (i.e., shorter reaction times), rather than risk-taking (i.e., more pumps). Importantly, we found that boredom proneness was correlated with reduced feedback processing as indexed by the feedback-P3. In the current study, high boredom prone individuals may benefit less from feedback precisely because they attend to it less and as a consequence are less sensitive to the payoff structure of the task (Kılıç et al. 2020). The feedback-P3 data certainly suggests that high boredom prone individuals demonstrate reduced feedback processing.

It could be that this decreased sensitivity to feedback is expressed as risk-taking in certain tasks, but not in the BART used here. Note that the BART requires an estimation of the average popping point, based on a partial-feedback scheme in which full feedback (i.e., the exact popping point of the balloon) is provided only when the balloon explodes. Thus, the BART is rather a complex decision-making under uncertainty task in which information seeking is confounded with risk-taking, potentially reducing the effect of either state boredom or boredom proneness on risk-taking.

It stands to reason that when participants show reduced attention and blunted feedback processing, their decisionmaking process will be inherently more noisy. This account, which we term here as noisy decision-making, could be erroneously labeled as risk-taking (Kılıç et al. 2020). Take for example a two-alternative forced-choice gambling task in which one of the alternatives is risky. Participants who benefit from feedback may learn to avoid the risky alternative (i.e., choose the risky option less than $50 \%$ of the time). Noisy decision-making, however, might be expected to approach chance, as participants are not adopting any particular decision-making strategy or preference. Thus, participants who alternated more between choices (i.e., choose the risky option approximately $50 \%$ of the time), may appear as more risk-seeking simply because their random behavior counteracts risk-aversion.

It is noteworthy that even in those studies we cited in the introduction investigating state boredom and risk-taking, the empirical findings are in line with our noisy decisionmaking hypothesis. In Kılıç et al. (2020) Study 3, it can be seen in their Fig. 3 that participants were overall risk-averse. That is, they preferred the safe alternative over the risky one. However, boredom pushed their choice rate towards 50\%

\footnotetext{
2 The one study that deviated from this pattern is Miao and colleagues' study 2, with an important difference between this study and the previous two: participants were not rewarded according to their performance. Previous studies show that when rewarding participants
}

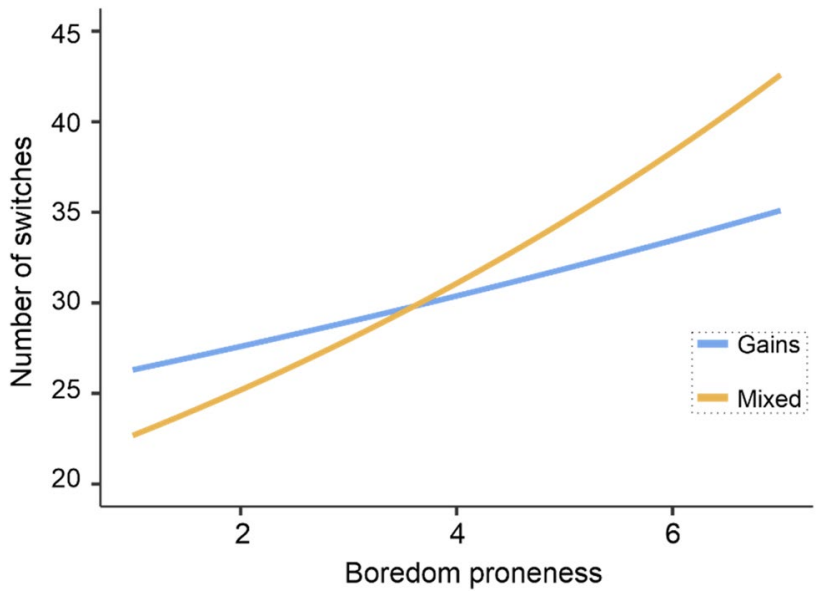

Fig. 3 Relationship between boredom proneness and decision switches in the two different reward conditions

(i.e., what would be expected by random choice). In Miao and colleagues' study 4 , participants in the low boredom condition showed risk-aversion $\left(P_{\text {risky }}=40 \%\right)$ but not in the high boredom condition $\left(P_{\text {risky }}=51 \%\right)$. These findings can be interpreted as increased risk-taking with boredom, but they could also be interpreted as noisy decision-making - leading to alternating between choices. ${ }^{2}$

\section{Study $2 a$-risk taking and choice switching in a simple decision-making task}

To address the question of whether boredom proneness is best characterized by noisy decision-making or risktaking, we adopted a widely used, simple decision-making from experience paradigm (Hertwig 2009). In this task participants repeatedly choose between two unmarked gambles and receive feedback on the obtained and foregone payoffs on each trial (i.e., full feedback). Participants do not receive any information on the distribution or possible outcomes of the gambles prior to the task, and they must therefore learn from experience (i.e., trial and error). Importantly, this task allows for the measurement of reliable individual differences in risk preferences and information seeking behaviors when partial feedback is provided (i.e., when feedback is given only for the chosen gamble;

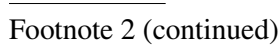

with real monetary payoffs (vs. hypothetical money), they are more sensitive to feedback (i.e., they adjust their behavior according to the outcomes of the previous trial), and when this reward is larger - they tend to be risk-averse (Xu, Xiao, \& Rao, 2019). 
Yechiam 2020), as well as choice switching (Yechiam 2015). In the partial-feedback paradigm, however, choice switching (i.e., alternating one's preference between prospects) is confounded with information seeking (i.e., exploration). Thus, here we used a full-feedback paradigm in which choice switching does not entail new information and allowed participants to sample each prospect as many times as they wished without monetary consequences, before making actual decisions. This design allowed us to disentangle change of preference from exploration, as participants were able to explore the gambles beforehand. Moreover, we included a mixed valence condition in which both gains and losses were possible - reflecting the fact that real-life risk-taking involves the possibility of losing (e.g., time, money or health). There is a well-documented asymmetry between gains and losses such that losses are typically weighted more than gains (Kahneman Tversky 1979). Thus, we expected participants to show preference for the safe gamble in the presence of losses. With respect to boredom proneness, if highly boredom prone individuals are less sensitive to the reward structure of the task then we expect they will not modulate their decision making based on the presence or absence of losses.

We hypothesized that (1) boredom proneness would be positively correlated with the number of trial-to-trial choice alternations in the decision-making task (i.e., noisy decisionmaking), and that (2) these alternations would lead to behavior that mimics risk-taking behavior. That is, insensitivity to reward structure will mean that with higher boredom proneness, individuals will choose more risky options.

\section{Participants}

Four hundred and thirty-nine participants were recruited via Amazon Mechanical Turk. They were offered \$1 CAD for completing the experiment, and a bonus ranging from $\$ 0$ to $\$ 1$ contingent on the amount of points they earned in the task ( 4 points $=1$ cent $)$. They were shown a short introduction form in which one line instructed them to input the word "thanks" in the comment section below. This procedure served to screen participants who did not read or comprehend the instructions (i.e., attention check) and has been successfully used in similar settings (Yakobi 2020). Our final sample included 404 participants (246 males, 153 females and five who chose not to disclose), aged from 20 to $71(M=37.2, \mathrm{SD}=10.8)$.

\section{Procedure}

Participants electronically signed a consent form, followed by a short general instructions page. This page included the attention check describe above. They were randomly assigned to the Mixed $(N=204)$ or Gains $(N=200)$ reward condition,
Table 2 Description of the gambles in each condition

\begin{tabular}{llllll}
\hline \multirow{2}{*}{ Condition } & \multicolumn{2}{l}{ Mixed domain } & & \multicolumn{2}{l}{ Gain domain } \\
\cline { 2 - 3 } \cline { 5 - 6 } \cline { 5 - 6 } & Safe & Risky & & Safe & Risky \\
\hline & $\sim \mathrm{U}(-1,6)^{\mathrm{a}}$ & $\sim \mathrm{U}(-5,10)$ & & $\sim \mathrm{U}(4,13)$ & $\sim \mathrm{U}(1,16)$ \\
& $\mathrm{EV}=2.5$ & $\mathrm{EV}=2.5$ & & $\mathrm{EV}=8.5$ & $\mathrm{EV}=8.5$ \\
\hline
\end{tabular}

EV Expected value

${ }^{\mathrm{a}}$ Uniform distribution (min, max)

then completed the short boredom proneness questionnaire and continued to the decision-making task. Upon completion of the decision-making task, participants reported how boring they perceived the task to be.

\section{Materials}

\section{Decision-making task}

The task was a basic binary gambling game in which two gambles (buttons) appeared on the screen from which participants could draw numbers from the respective distributions. One of the gambles was associated with a safe distribution (low variance) and the other with a risky distribution (high variance; for the complete description of the distributions Table 2). In the Gains condition, both safe and risky gambles yielded only positive numbers, while in the mixed condition the outcome distributions included losses. The task started with a sampling stage in which participants were encouraged to sample each gamble as many times as they wanted (but at least once from each gamble), and were provided with partial feedback (i.e., each sample generated one random outcome from the respective gamble). They were instructed that the sampling stage has no effect on their final payoff and that it simply served to allow them to study the gambles. When they were done sampling, the decision stage began. The points they gained (or lost) in the 100 trials of the decision stage determined their final bonus. In the decision stage participants were given full feedback (see Appendix B for the full instructions and screenshots of the task). The position of the two buttons was counterbalanced across participants. We calculated three dependent variables; (1) the number of samples drawn in the sampling stage, (2) the number of switches made in the decision stage, and (3) the number of risky choices made in the decision stage.

\section{State boredom}

Upon task completion, we asked participants to rate how boring was the decision-making task on a 1 (not boring at all) to 9 (highly boring) Likert scale. 
Table 3 Descriptive statistics of the variables in Study 2a

\begin{tabular}{lcllllc}
\hline & \multicolumn{2}{l}{ Condition } & & \\
\cline { 2 - 3 } & \multicolumn{2}{l}{ Gains } & & & \multicolumn{2}{l}{ Mixed } \\
\cline { 2 - 3 } \cline { 5 - 6 } \cline { 5 - 6 } & Mean & SD & & Mean & SD \\
\hline Boredom proneness & 3.039 & 1.496 & & 2.865 & 1.376 \\
State boredom & 5.045 & 2.501 & & 4.708 & 2.445 \\
Samples & 16.540 & 15.084 & & 17.966 & 19.157 \\
Proportion of samples switches & 0.450 & 0.295 & & 0.453 & 0.310 \\
Number of risky decisions & 49.5 & 23.2 & & $45.1 * *$ & 27.4 \\
Boredom proneness & 3.039 & 1.496 & & 2.865 & 1.376 \\
State boredom & 5.045 & 2.501 & & 4.708 & 2.445 \\
\hline
\end{tabular}

**Different from $50, p<0.005$

\section{Short boredom proneness scale (sBPS)}

We used the same scale as in Study 1, which showed excellent internal consistency in this sample (Cronbach's $\alpha=0.925$ ).

\section{Results}

State and trait boredom ratings were similar to those reported in Study $1\left(\mathrm{M}_{\text {boredom-proneness }}=2.95, \mathrm{SD}=1.44\right.$; $\mathrm{M}_{\text {state-boredom }}=4.88, \mathrm{SD}=2.48$ ) and were not significantly different between the two groups (Tables 3, 4 for a correlation matrix).

Decision switches and boredom. To test our first hypothesis that boredom proneness would be related to noisy decision-making, we calculated the number of switches for each participant in the decision stage (ranging 0-99). We entered this count into a Poisson regression as the dependent variable, with condition and boredom proneness as independent variables. We used GAMLj package with JAMOVI (The JAMOVI project, 2020) for this analysis, and corrected for overdispersion since the dispersion index was high (Value/df $=13.646)$, suggesting high overdispersion ${ }^{3}$ in our data. We found a significant effect of boredom proneness $\left(\exp (\mathrm{B})=1.08, \chi^{2}=10.51, \mathrm{p}=0.001\right)$ but not for condition $\left(\exp (\mathrm{B})=0.815, \chi^{2}=1.62, p=0.203\right)$ or their interaction $\left(\exp (\mathrm{B})=1.059, \chi^{2}=1.48, p=0.223\right)$. This suggests that regardless of the condition, for every unit of boredom proneness there was an increase of $8 \%$ in the number of switches (Fig. 3).

\section{Risky behavior and boredom}

We used the same overdispersion Poisson model with the number of risky choices (defined as the number of trials a participant chose the risky gamble) as the dependent variable. There was a significant effect of condition $\left(\exp \left(\mathrm{B}_{\text {mixed }}\right)=0.776, \chi^{2}=4.07, p=0.044\right)$ indicating that participants chose the risky option more in the gains condition compared with the mixed condition. There was no significant effect of boredom proneness $(\exp (\mathrm{B})=1.019$, $\left.\chi^{2}=1.03, p=0.311\right)$ or their interaction $(\exp (\mathrm{B})=1.054$, $\chi^{2}=1.9, p=0.168$ ) on overall risk-taking. As can be seen in the top left panel of Fig. 4, there is an apparent increase in risk-taking with experience in low boredom prone individuals over time in the mixed gains condition, thus we focus next on the first 25 trials (first block) where risk-aversion was highest. When considering the number of risky choices in the first block, there are significant effects of boredom proneness $\left(\exp (\mathrm{B})=1.046, \chi^{2}=4.59, p=0.032\right)$, condition $\left(\exp (\mathrm{B})=0.699, \chi^{2}=6.39, \mathrm{p}=0.012\right)$, and a marginal interaction effect $\left(\exp (\mathrm{B})=1.081, \chi^{2}=3.48, p=0.062\right)$. Simple slopes analysis indicated that boredom proneness predicts risky behavior in the mixed domain $(\exp (\mathrm{B})=1.09$, $\left.\chi^{2}=7.313, p=0.007\right)$, but not in the gains domain $\left(\exp (\mathrm{B})=1.01, \chi^{2}=0.047, p=0.828 ;\right.$ Fig. 4 , right panel $)$.

\section{Sampling and boredom}

There was no significant effect of boredom proneness or condition on the number of samples drawn in the sampling stage.

\section{Exploratory analysis}

Next, we explored the notion that decision switching is a mechanism for coping with boredom. That is, high boredom prone individuals tend to alternate their gambling decisions more to alleviate feelings of state boredom. Using the MEDMOD plug-in in JAMOVI, we tested a mediation model in which decision switching mediates the relationship between boredom proneness and state boredom. The mediation model was significant $(\beta=0.281, p<0.001)$, with both direct and indirect effects $(\beta=0.282, p<0.001)$. As can be seen in Fig. 5, boredom proneness is positively and directly associated with state boredom, but the indirect relationship, via decision switching, is negative. Thus, the more boredom prone individuals are, the more boredom they tend to experience in the task; this relationship, however, is suppressed when decision switches are high.

\footnotetext{
3 Overdispersion describes a distribution with higher than expected variance (Gardner et al. 1995).
} 
Table 4 Correlation matrix of the variables in Study $2 \mathrm{a}$

\begin{tabular}{llcllll}
\hline & 1 & 2 & 3 & 4 & 5 & 6 \\
\hline 2 & State boredom & $0.282^{* * *}$ & - & & & \\
3 & Num. of samples & -0.021 & -0.012 & - & & \\
4 & Sample switches & $0.111^{*}$ & 0.04 & $-0.399 * * *$ & - & \\
5 & P(risky) & 0.047 & 0.013 & $0.105^{*}$ & 0.086 & - \\
6 & Decision switches & $0.159^{* *}$ & $0.128^{*}$ & $0.317^{* * *}$ & $0.264^{* * *}$ & $0.109^{*}$ \\
\hline
\end{tabular}
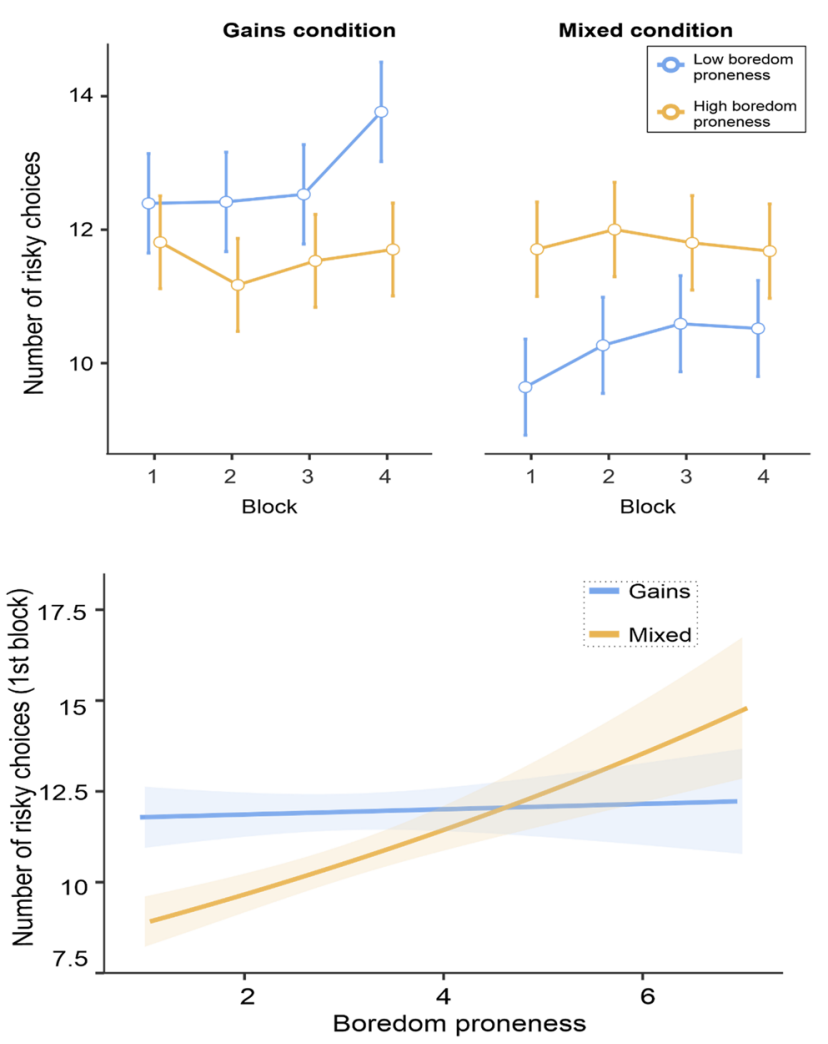

Fig. 4 Top: The number of risky choices by blocks of 25 trials, condition and boredom proneness (median split for illustration). Error bars represent standard errors. Bottom: The number of risky choices in the first block, by condition and boredom proneness

\section{Summary and discussion}

In Study $2 \mathrm{a}$ we showed that boredom proneness positively predicts choice switching between the two gambles in the decision-making task: individuals high on trait boredom proneness switched between gambles more often than those low in trait boredom proneness. A reasonable alternative interpretation to choice switching, would be that high boredom prone individuals explore more, as they seek information and novelty. This claim, however, is not supported by the data: first, participants received feedback on both

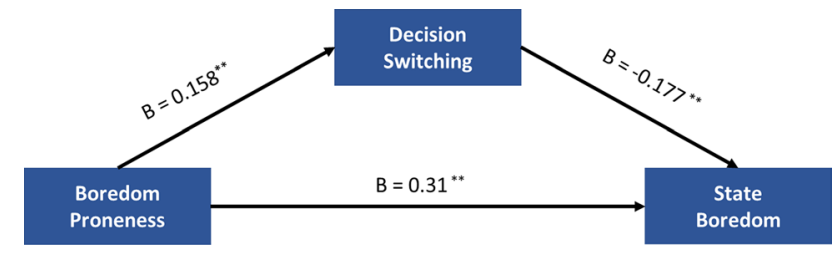

Fig. 5 Mediation model with standardized regression coefficients. $* *<0.001$

obtained and foregone payoffs, hence switching does not provide additional information. Second, if high boredom prone individuals explore more, one would expect more sampling in the sampling stage, which we did not find.

Interestingly, in the first block, before learning had occurred, choice switching translated into a positive relationship between boredom proneness and risky behavior. As we hypothesized, this relationship was more pronounced in the presence of losses (i.e., in the Mixed condition). However, as can be seen in the left panel of Fig. 4, this is most likely due to low boredom prone individuals changing their choice preference between the two conditions, while high boredom prone individuals do not, making choices that are seemingly oblivious to the reward structure. Lastly, an exploratory analysis showed that switching behavior may serve as a mechanism to cope with boredom induced by the task.

\section{Study $\mathbf{2 b}-$ pre-registered replication of study $2 a$}

Study 2 a showed increased choice switching with boredom proneness, but further exploration of the data showed increased risk-taking with boredom proneness in the first block, and that choice switching alleviated in-the-moment feelings of boredom in the high boredom prone individuals. Due to the exploratory nature of the latter two findings, here we conducted a replication study. A common finding in the boredom literature is that high boredom prone individuals exhibit lower levels of self-control (Boylan et al. 2020; Isacescu Danckert 2018; Struk et al. 2016; Wolff 
Martarelli 2020; Wolff et al. 2020), with correlations as high as $r=-0.6$. As such, here we also measured and controlled for trait levels self-control. This direct replication was preregistered at https://osf.io/gqbax.

\section{Participants}

Four hundred and thirty-one participants were recruited via Amazon Mechanical Turk. They were offered \$1.5 CAD for completing the experiment, and a bonus ranging from $\$ 0$ to $\$ 1$ contingent on the number of points they earned in the task ( 4 points $=1$ cent). The same screening procedure and criteria were used as in Study 2a. Our final sample included 420 participants ( 217 males, 201 females and two who chose not to disclose), aged from 18 to $75(M=39.74, \mathrm{SD}=11.36)$.

\section{Procedure and materials}

The procedure and materials were identical to Study 2a, with the exception that participants also performed the Iowa Gambling Task (which we report in Study 3) prior to, or after the task. In addition, we added the Brief Self-Control Scale (BSCS; Tangney et al. 2004) after the short boredom proneness scale. The BSCS includes 13 items on a 1-5 Likert scale, such as "I often act without thinking through all the alternatives" and "I do certain things that are bad for me if they are fun". We used the same statistical tests and measures as we did in Study 2a.

\section{Results}

There were no significant differences between the two experimental groups (i.e., gains only and mixed rewards) in any of the study's variables (Table 5 for descriptive statistics and Table 6 for a correlation matrix).
Table 5 Descriptive statistics of the variables in Study $2 b$

\begin{tabular}{lccllc}
\hline & \multicolumn{2}{l}{ Condition } & & \\
\cline { 2 - 3 } & \multicolumn{2}{l}{ Gains } & & & Mixed \\
\cline { 2 - 3 } \cline { 5 - 6 } \cline { 5 - 6 } & Mean & SD & & Mean & SD \\
\hline Boredom proneness & 2.87 & 1.37 & & 2.66 & 1.29 \\
Self-control & 3.47 & 0.81 & & 3.53 & 0.78 \\
State boredom & 4.5 & 2.68 & & 4.48 & 2.7 \\
Samples & 20.13 & 17.84 & & 21 & 21.56 \\
Proportion of samples switches & 0.382 & 0.3 & & 0.348 & 0.27 \\
Number of decision switches & 25.43 & 20.56 & & 23.76 & 19.9 \\
Number of risky decisions & 53.48 & 26 & & 46 & 28.6 \\
\hline
\end{tabular}

\section{Decision switches and boredom}

We modeled our data using an overdispersion Poisson model just as we did in Study 2a, but added self-control as a covariate. This analysis replicates the results of Study $2 \mathrm{a}$, with boredom proneness, but not self-control or condition, positively predicting the amount of decision switching $\left(\exp (\mathrm{B})=1.13, \chi^{2}=10.34, p=0.001\right)$. There were no significant interaction effects.

\section{Risky decision-making and boredom}

Similar to Study $2 \mathrm{a}$, we found risk-aversion in the mixed domain $\left(\exp \left(\mathrm{B}_{\text {mixed }}\right)=0.865, \chi^{2}=7.133, p=0.008\right)$, and no relationship between risky decision-making and boredom proneness. There was no significant interaction effect, and self-control did not affect risk-taking $\left(\exp \left(\mathrm{B}_{\mathrm{sc}}\right)=1.003\right.$, $\chi^{2}=0.005, p=0.94$ ). Unfortunately, we were unable to replicate the interaction between condition and boredom proneness in the first block found in Study 2a $(p=0.956)$.

\section{Mediation}

As we found in Study 2a, decision switching partially mediated the relationship between boredom proneness and state
Table 6 Correlation matrix of the variables in study $2 b$

\begin{tabular}{lllllllll}
\hline & 1 & 2 & 3 & 4 & 5 & 6 & 7 \\
\hline 2 & State boredom & $0.291 * * *$ & - & & & & & \\
3 & Self-control & $-0.622 * * *$ & $-0.3 * * *$ & - & & & & \\
4 & Num. of samples & -0.092 & -0.018 & 0.036 & - & & & \\
5 & Sample switches & $0.146 * *$ & -0.029 & -0.009 & $-0.447 * *$ & - & & \\
6 & P(risky) & -0.022 & -0.067 & 0.02 & -0.011 & 0.019 & - & \\
7 & Decision switches & $0.146 * *$ & $-0.178^{* * *}$ & -0.034 & $-0.265^{* * *}$ & $0.408^{* * *}$ & 0.028 & - \\
\hline$* p<0.05, * * p<0.01, * * * p<0.001$ & & & & & &
\end{tabular}




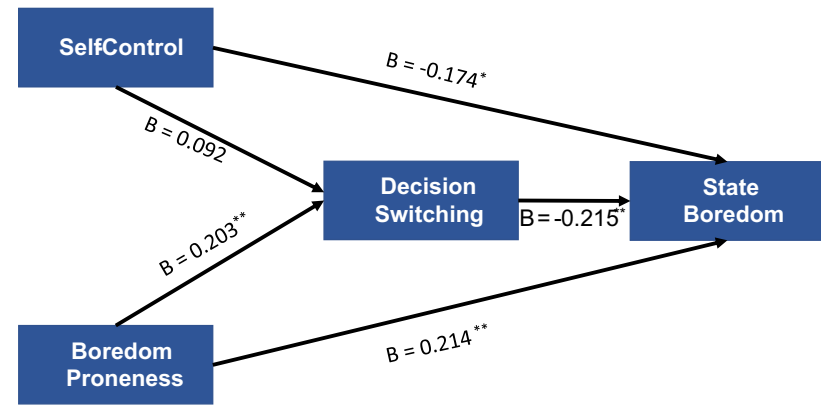

Fig. 6 Mediation model with standardized regression coefficients. ${ }^{*}<0.01,{ }^{* *}<0.001$

boredom, even in the presence of self-control. There was a significant direct, positive relationship between boredom proneness and state boredom $(\beta=0.214, p<0.001)$, and an indirect, negative relationship $(\beta=-0.152, p<0.001)$. As can be seen in Fig. 6, high self-control predicted lower levels of in-the-moment feelings of boredom, but it did not correlate with decision switching.

\section{Summary and discussion}

We replicated the main findings of Study 2a: with higher boredom proneness, individuals tend to switch more between decisions, beyond any effect of self-control. This switching behavior, in turn, attenuates the experienced levels of state boredom.

\section{Study 3-Decision making and feedback sensitivity in the lowa Gambling Task}

In study 1, we showed neural evidence for reduced feedback processing with higher boredom proneness and in studies $2 \mathrm{a}$ and $2 \mathrm{~b}$, we showed its behavioral expression-increased choice switching. One way to bridge the behavioral data and purported cognitive mechanism is using computational modeling - a popular way to address the cognitive mechanisms underlying decision-making (Farrell Lewandowsky 2018). We chose to model behavior using the Iowa Gambling Task
(IGT; Bechara et al. 1994), a decision-making task that is extensively used to study individual differences in decisionmaking and as in the previous tasks used here, a task that relies exclusively on feedback. Studies using the IGT have shown before reliable individual differences related to trait anxiety, as well as symptoms of schizophrenia (Miu et al. 2008; Shurman 2005). Behavioral data and computational modeling also show relationship with age, such that decision-making performance peaks in adulthood, compared with performance in children and older adults (Beitz 2014; Hooper et al. 2004).

\section{Participants}

Participants in this study were 413 individuals, out of the 420 participants that we recruited via Mechanical Turk for Study 2b. There were 214 males, 197 females and two who chose not to disclose, aged from 18 to 75 years old $(M=39.62, \mathrm{SD}=11.17)$.

\section{Procedure and materials}

The procedure is described in Study 2b. Briefly, participants filled out the short boredom proneness scale and a brief self-control scale, and then performed the IGT and the task reported in Study 2b, in a counterbalanced order. Upon completion, they reported the level of boredom they experienced on a 1-9 scale.

\section{The lowa Gambling Task (IGT)}

In the IGT, participants are given an initial loan of $\$ 2000$ play money and are presented with four decks of cards. They are instructed to maximize their payoff during the 100 consecutive deck selections, such that $\$ 40$ in play money will be converted to \$1 USD. After each choice, they receive feedback on the chosen (obtained) outcomes which always entail gains, but sometimes also incur losses (Table 7). There are two good decks (C, D) which yield a gain of \$25 in the longterm, and two bad decks which result in a loss of $\$ 25$ in the long-term (A,B). Decks B and D are considered riskier as
Table 7 Description of the card decks in the Iowa Gambling Task

\begin{tabular}{|c|c|c|c|c|}
\hline & Deck A & Deck B & Deck C & Deck D \\
\hline Reward & $\$ 100$ & $\$ 100$ & $\$ 50$ & $\$ 50$ \\
\hline Penalty & $\begin{array}{l}\text { Frequent (50\%) \$150- } \\
\quad \$ 350\end{array}$ & $\begin{array}{l}\text { Infrequent }(10 \%) \\
\$ 1250\end{array}$ & $\begin{array}{l}\text { Frequent }(50 \%) \\
\quad \$ 25-\$ 75\end{array}$ & $\begin{array}{c}\text { Infrequent } \\
(1 \%) \\
\$ 250\end{array}$ \\
\hline Long-term payoff & $-\$ 25$ & $-\$ 25$ & $+\$ 25$ & $+\$ 25$ \\
\hline
\end{tabular}




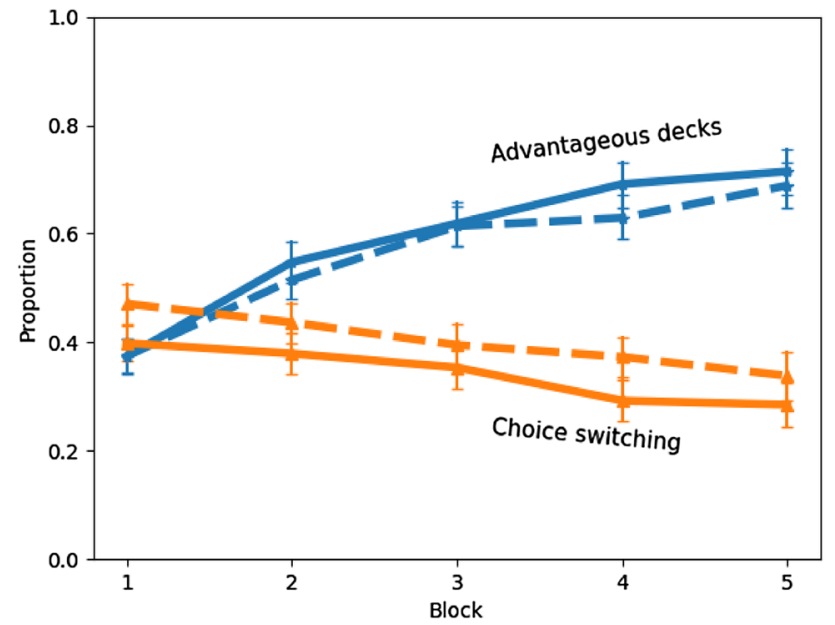

Fig. 7 Advantageous choice and switching rates in blocks of 20 trials (error bars represent 95\% CI). Dash lines: high boredom proneness, Solid lines: low boredom proneness

their associated losses are higher and infrequent. The order of the four decks was counterbalanced between participants.

\section{Results}

\section{Behavior}

First, we present the overall preferences and switching throughout the task (Fig. 7). Consistent with previous studies, participants learn to choose the advantageous decks (C,D) over the bad ones, and switch between decks less often with experience.

To test the associations between boredom proneness, state boredom, and behavior in the IGT, we ran three overdispersion Poisson regression models as in studies $2 \mathrm{a}$ and $2 \mathrm{~b}$, predicting the number of advantageous decks decisions, number of risky decisions, and the number of choice switches, controlling for self-control.
As can be seen in Table 6, Boredom proneness positively predicted the number of choice switches, which was the only significant effect. There was, however, a marginally significant $(p=0.06)$ effect of boredom proneness on risky behavior, such that risky behavior was reduced with increasing levels of boredom proneness (i.e., the opposite effect relative to literature on boredom proneness and self-reported risk-taking) (Table 8).

\section{Computational modeling}

We adopted the expectancy valence model (EVM; Busemeyer Stout 2002) - an extensively used model for capturing individual differences in the IGT. Here we adopted a more recent version of the EVM, the two-parameter EVM (2pEVM; Humphries et al. 2015), which was shown to have better performance in capturing psychological processes in individuals. In the EVM, the utility $u$ for each outcome is calculated with $0<\mathrm{w}<1$ as a free parameter representing the weight given to losses.

$u_{k}(t)=(1-w) \times \operatorname{Gain}(t)+w \times \operatorname{Loss}(t)$

The model assumes that in each trial, players update their expectancy $E v$ in a given trial $t+l$ for the selected deck $k$, by integrating feedback (outcome $U_{k}$ ) with their previous experience, where $0<a<1$ is the updating rate parameter (higher values suggest higher weight for more recent feedback).

$E v_{k}(t+1)=(1-a) \times E v_{k}(t)+a \times U_{k}(t)$

After an initial random choice, the probability of choosing deck $k$ in trial $t+1$ is determined by a softmax rule (Luce, 1959), such that the probability for choosing deck $D$ is formulated as

$P\left[D_{i, t+1}\right]=\frac{e^{E v_{i, t} * \theta_{(t)}}}{\sum_{j=1}^{4} e^{E v_{j, t} * \theta_{(t)}}}$
Table 8 Effects of boredom proneness, state boredom and self-control on behavior in the IGT

\begin{tabular}{llll}
\hline & Choices Switching & Advantageous choices & Risky choices \\
\hline Boredom proneness & $\operatorname{Exp}(\mathrm{B})=1.036$ & $\operatorname{Exp}(\mathrm{B})=0.97$ & $\operatorname{Exp}(\mathrm{B})=0.968$ \\
& $95 \%$ CI $[1.036-1.17]$ & $95 \%$ CI $[0.937-1]$ & $95 \%$ CI [0.936 1] \\
& $\chi^{2}=9.58, p=0.002$ & $\chi^{2}=2.849, p=0.093$ & $\chi^{2}=3.548, p=0.06$ \\
State boredom & $\operatorname{Exp}(\mathrm{B})=0.997$ & $\operatorname{Exp}(\mathrm{B})=0.988$ & $\operatorname{Exp}(\mathrm{B})=0.999$ \\
& $95 \%$ CI $[0.974-1.02]$ & $95 \%$ CI $[0.9751]$ & $95 \%$ CI $[0.9871 .01]$ \\
Self-control & $\chi^{2}=0.046, p=0.83$ & $\chi^{2}=2.93, p=0.088$ & $\chi^{2}=0.011, p=.914$ \\
& $\operatorname{Exp}(\mathrm{B})=1.052$ & $\operatorname{Exp}(\mathrm{B})=0.975$ & $\operatorname{Exp}(\mathrm{B})=0.982$ \\
& $95 \%$ CI $[0.95-1.17]$ & $95 \%$ CI $[0.921-1.03]$ & $95 \%$ CI $[0.929-1.04]$ \\
& $\chi^{2}=0.937, p=0.335$ & $\chi^{2}=0.789, p=0.374$ & $\chi^{2}=0.43, p=.512$ \\
\hline
\end{tabular}


The free parameter $0<\theta<1$ reflects the sensitivity to expectancy (low values lead to more stochastic behavior). In the classical EVM, $\theta$ is trial-dependent, such that with experience, the decision process becomes less random

$\theta(t)=\left(\frac{t}{10}\right)^{c}$

In the $2 \mathrm{pEVM}$, the free parameter $c$ is held constant at 0.5 , leaving the number of free parameters at two $(w, a)$. We used maximum likelihood estimation to fit the two parameters for each participant, on a single trial level. A simplex algorithm was used for maximizing the log-likelihood function (Nelder Mead 1965).

The zero-order correlation between the observed and predicted proportion of advantageous deck choices was $r=0.89$, suggesting a good fit with the data. We then regressed each of the model's parameters, namely $w$ (loss/gain weight) and $a$ (updating rate) on boredom proneness, state boredom and self-control. The model predicting $a$ was significant $\left(R^{2}=0.019, \mathrm{~F}(3,409)=2.69, p=0.046\right)$, suggesting that with higher boredom proneness levels, participants updating rate decreases $(\beta=-0.14, p=0.026)$. There were no significant effects for state boredom $(\beta=-0.02, p=0.682)$ nor selfcontrol $(\beta=-0.012, p=0.842)$. The model predicting $w$ was not significant $\left(R^{2}=0.009, \mathrm{~F}(3,409)=1.36, p=0.256\right)$.

\section{General discussion}

In the present work, we aimed to investigate the relationship between boredom proneness and risky decision making in the lab. In contrast to previous studies building on selfreports, we found no evidence that boredom proneness is associated with risky decision making in three widely studied decision-making tasks. We then proposed that reduced feedback processing, leading to noisy decision making, may be an important contributor to the purported boredom-risk taking relationship, a factor that has largely been overlooked in the literature. This notion is based on a large research base showing that boredom proneness is linked with attention deficits (Eastwood et al. 2012; Hsu et al. 2020; Hunter Eastwood 2018; Isacescu et al. 2017; Malkovsky et al. 2012). Indeed, we found reduced sensitivity to feedback and a robust boredom proneness-choice switching relationship, even when controlling for trait self-control. To our knowledge, this is the first study to demonstrate that boredom proneness is associated with increased choice switching and decreased sensitivity to feedback, as well as the first to empirically challenge its relationship with risk-taking.

We investigated the neural correlates of risky decision making in the context of boredom and boredom proneness using the BART - a well-validated task, often used to assess individual differences in risk-taking. We found that the FRN was not significantly correlated with boredom proneness. It has been previously demonstrated that the FRN and feedback-P3 represent different aspects of the feedback signal (Yeung Sanfey 2004). A double dissociation between these two components suggests that the FRN is sensitive to valence (i.e., gains versus losses) while the feedback-P3 represents reward magnitude (Yeung Sanfey 2004). In the BART we used here there were no losses, only gains: on each trial, participants either gained the number of pumps they chose or did not gain anything if the balloon exploded. In that context, we found reduced feedback-P3 amplitudes with higher levels of boredom proneness, suggesting that individuals who tend to experience boredom more frequently and intensely, are less sensitive to feedback.

We did not find behavioral evidence for risk-taking with higher boredom proneness. It is possible that we did not find this relationship in the BART due to the lack of losses in the specific variation of the task we used here. That is, high boredom prone individuals may be risk-seeking only in environments that entail losses. In addition, the BART could be considered a more complex learning task, as the optimal strategy requires estimating the mean of an unknown uniform distribution. We tested these two possible explanations by contrasting a gains only domain (as in the BART) with mixed valence domain, in a binary decision task (studies $2 \mathrm{a}$ and $2 b$ ). We did find an association between boredom proneness and risk-taking in the first block in the mixed domain condition (study $2 \mathrm{a}$ ), but due to the nature of this exploratory analysis, the fact that we could not replicate it in study $2 b$ and study 3, we conclude that there is no evidence for risktaking with boredom proneness in tasks used here.

We build on the finding of reduced feedback sensitivity, together with the literature linking boredom proneness with poor attentional control (refs), to argue that boredom proneness is associated with noisy decision-making. We chose to term the behavior and proposed mechanism "noisy decisionmaking" for two reasons. First, insensitivity to feedback, and consequently to the reward structure of the task, means that individuals base their decisions less on relevant information (i.e., the utility or value of the given options), and on factors that are unrelated to the reward structure. While this is not completely random as might be implied by "noisy decision making", it does seem to us to be impoverished relative to an optimal strategy, and we are attempting to capture that impoverishment with the term "noisy". Second, this somewhat ambiguous term (e.g., compared with "random decision-making") reflects that we are, to some extent, more confident in our behavioral findings, than in the mechanism we propose (i.e., reduced sensitivity to feedback). Thus, we wish to leave room for future studies to directly test reduced 
feedback sensitivity as the driver of the boredom pronenessrisk-taking relationship, both in neurophysiology and behavior. Such studies could, for example, test whether high boredom prone individuals exhibit reduced feedback sensitivity in tasks that allow choice switching (as in studies $2 \mathrm{a}, \mathrm{b}$ and 3 ) by recording EEG and exploring whether this reduced sensitivity plays a role in choice switching.

Other factors may account for the association between boredom proneness and choice switching. For example, choice switching may act as a mean to gather new information (i.e., exploration), with choice switching functioning merely as one possible exploration strategy (Yechiam 2020). There are, however, two caveats for interpreting choice switching as exploration within the present work. First and most important, the task used in studies $2 \mathrm{a}$ and $2 \mathrm{~b}$ provide full feedback, thus there is no benefit for alternating between choices in terms of exploration. In other words, choice switching did not add more information. Second, previous work showed that the highly boredom prone tend to ruminate whereas exploratory behavior may better characterize individuals low in boredom proneness (Danckert 2019; Mugon et al. 2018). Another possibility is that those who are high in boredom proneness seek novelty and new sensations (Dahlen et al. 2004, 2005; Kass Vodanovich 1990). If that were the case, however, one would expect boredom proneness to be correlated with risk-taking, in study 1 (i.e., balloon popping as sensation seeking), and in studies $2 \mathrm{a}$ and $2 b$ (i.e., choosing the alternative with the wider uniform distribution, which yields novel outcomes more often). Although this is not the case in our data, we did not measure sensation seeking explicitly and future studies should test these alternative mechanisms.

It could be argued that even if the noisy decision-making hypothesis is fully supported in the lab, it does not account for real-life risky behavior (e.g., drug and alcohol abuse, risky sex behavior, rule breaking during the pandemic; Biolcati et al. 2018; Boylan et al. 2020), where incentives and feedback are not always immediately available. While we do not claim that noisy decision-making exclusively explains the relation between risky behavior and boredom proneness, however, it is an important aspect to consider when examining this relation outside the lab. In real-life, there are normally more ways to behave recklessly than there are ways to behave safely. Consider for example a simple task such as crossing a busy two-way road. There are plenty of risky ways to cross this road: with eyes closed, while texting, while walking backwards, crawling, while looking only to the left and so forth. These behaviors may seem senseless to most adults (but apparently not to all: Lennon et al. 2017). However, they are senseless only because we were taught and trained how to safely cross a busy road: being attentive, using all our available senses, looking to the right and left and only then crossing promptly in a straight line. Thus, our action space is full of dangerous prospects, but only a limited range of safe behaviors. Similarly, when one is eager for an engaging activity, one's action space is likely to include safe behaviors (e.g., reading the newspaper or meeting a friend), as well as behaviors that could be fulfilling in the short-term, yet harmful in the long run (e.g., smoking, drinking, street racing). If the decision-maker is not considering the implications of all options carefully, their behavior may seem somewhat arbitrary (i.e., less reliant on the utility or value of the given options). In other words, poor apprehension of the outcome space may masquerade as increased risk taking, when it is related to other factors such as poor attention to contingencies or deficient use of feedback.

State boredom has an important function in regulating behavior. As a cognitive-affective state, boredom is a call to action (Bench Lench 2013), signaling rising opportunity costs (Danckert 2019; Kurzban et al. 2013; Struk et al. 2020). Individuals who do not respond adaptively to this signal (e.g., individuals low in self-control; Isacescu Danckert 2018), also tend to be high in boredom proneness (Danckert et al. 2018; Isacescu et al. 2017). Previous studies show that individuals try to avoid the aversive experience of boredom, even by seeking negative experiences (Bench Lench 2019) or sadistic behaviors (e.g., killing worms; Pfattheicher et al. 2020). In support for this notion, the analysis in Study 2a (replicated in Study 2b), shows that people high in boredom proneness may regulate in-the-moment feelings of boredom by alternating between choices. While this can appear "risky" it may be better characterized as insensitivity to reward structures. A notable limitation of this analysis is the lack of an explicit state boredom mood induction. Arguably, other emotional states that were not measured and controlled for (e.g., frustration, motivation) could account for this finding, as well as the lack of a significant correlation between state boredom and risk-taking.

As noted by Miao and colleagues (2019), risk is a multifaceted construct, defined differently in different fields of study. In public health and psychology, risk is often defined with respect to the possibility of negative or harmful outcomes, while in behavioral economics alternatives with higher variability are considered riskier - a definition we implicitly adopted here. Future research could address this distinction, for example by investigating whether risk as outcome variability and risk as potentially harmful outcomes are differentially affected by both state boredom and boredom proneness. 
Finally, we would like to point out two more issues that should be considered when interpreting our results. First, although boredom proneness is discussed here as an antecedent of noisy decision-making, we cannot conclusively determine the causal direction of these relations. That is, although we controlled for self-control which is highly correlated with boredom proneness, other trait level variables or conditions (e.g., ADHD) could contribute to this association. In addition, one should note the uniqueness of the online sample in relation to boredom proneness. That is, participants in online data collection platforms such as Mechanical
Turk (i.e., individuals who choose to participate in highly repetitive and boring tasks) may have a different set of characteristics and strategies for coping with boredom. Although the mean boredom proneness is lower in the online samples (2.95 and 2.76) than in the student sample (3.08), this difference did not reach significance when formally analyzed (Appendix C).

\section{Appendix}

See Figs. 8, 9, 10.

Fig. 8 EEG Processing Pipeline

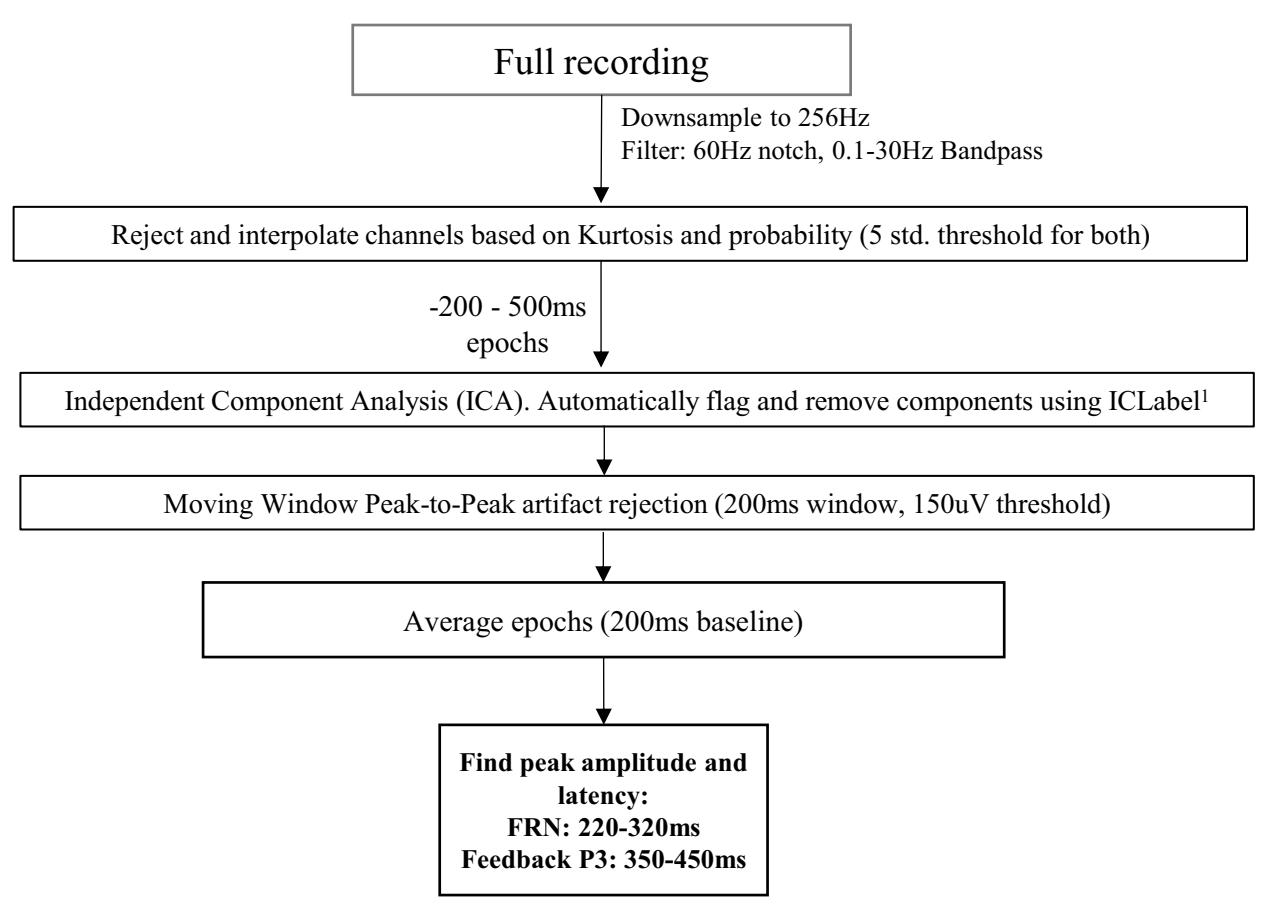




\section{Decision-making game}

In this game, you have to choose once, between two alternatives: "A" or "B".

Each alternative is associated with a specific distribution of outcomes, generated by the computer.

Once you choose an alternative (A or B), the computer randomly draws a number depending on the alternative you chose.

A

Positive numbers are gains - they are accumulated in your total earnings. Negative numbers are losses, they are deducted from your

earnings. Luckily, you can "sample" each alternative - as many times as you wish!

Sampling allows you to try out the two options without having an effect on your actual earnings.

After you are done sampling, you will continue to the decisions stage that determines your payoff from this HIT

\section{I understand}

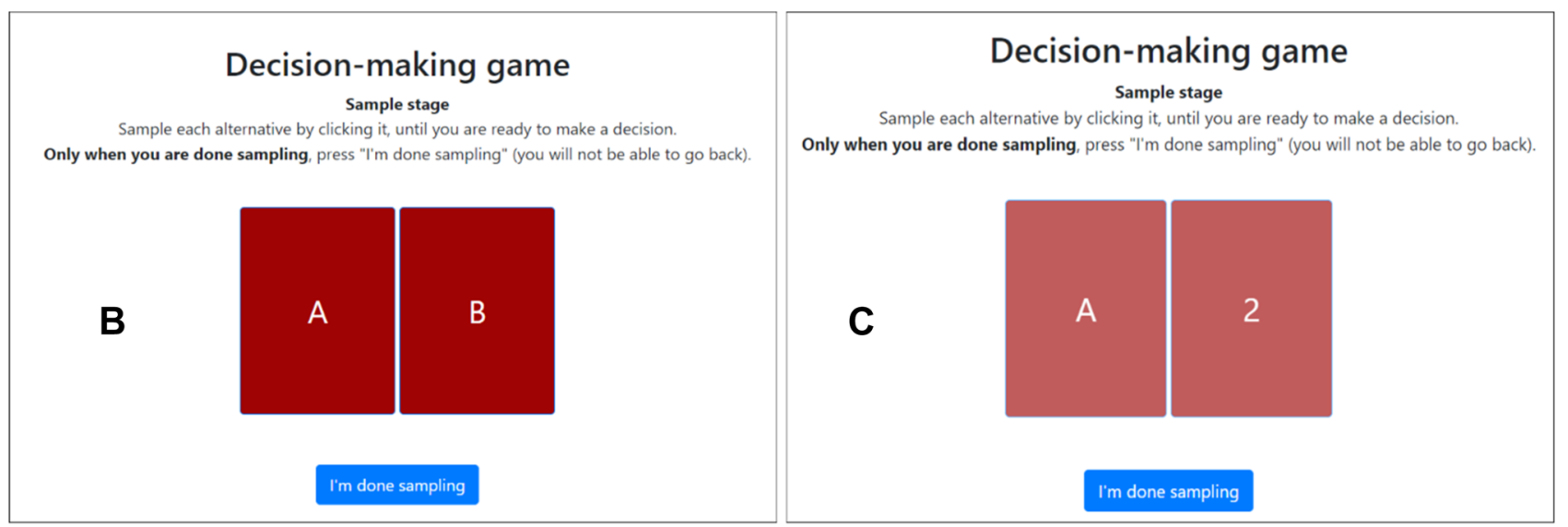

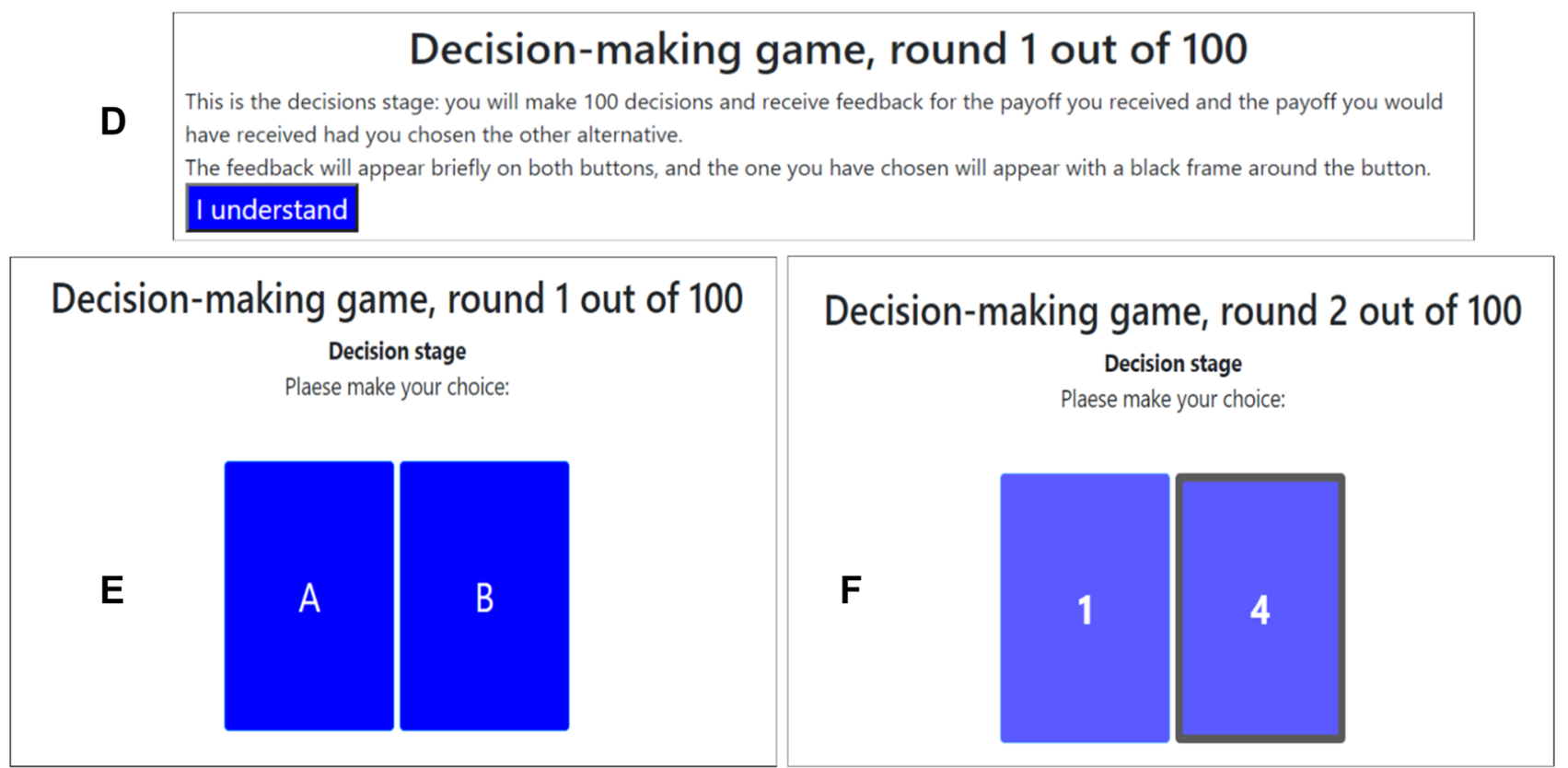

Fig. 9 Study 2 task's instructions and screenshots. a Instructions for the task, (b) Sampling, (c) Sampling—feedback, (d) Instructions for the decision stage, (e) Decision stage, and (f) Decision stage, and (e) Decision stage—feedback 
Fig. 10 Comparison of boredom proneness between studies' samples. The histograms above show that the mTurk samples (Studies 2a, 2b and 3) are well distributed across the boredom proneness scale. However, a repeated measure ANOVA suggests that there could be a significant effect of sample on the average levels of boredom proneness $(\mathrm{F}(2,263)=3.59$, $p=0.048$ ). A planned contrast analysis comparing the student sample (Study 1) with the two mTurk samples, however, did not show a significant difference between the online and students samples $(\mathrm{t}(117.1)=1.754$, $p=0.082)$
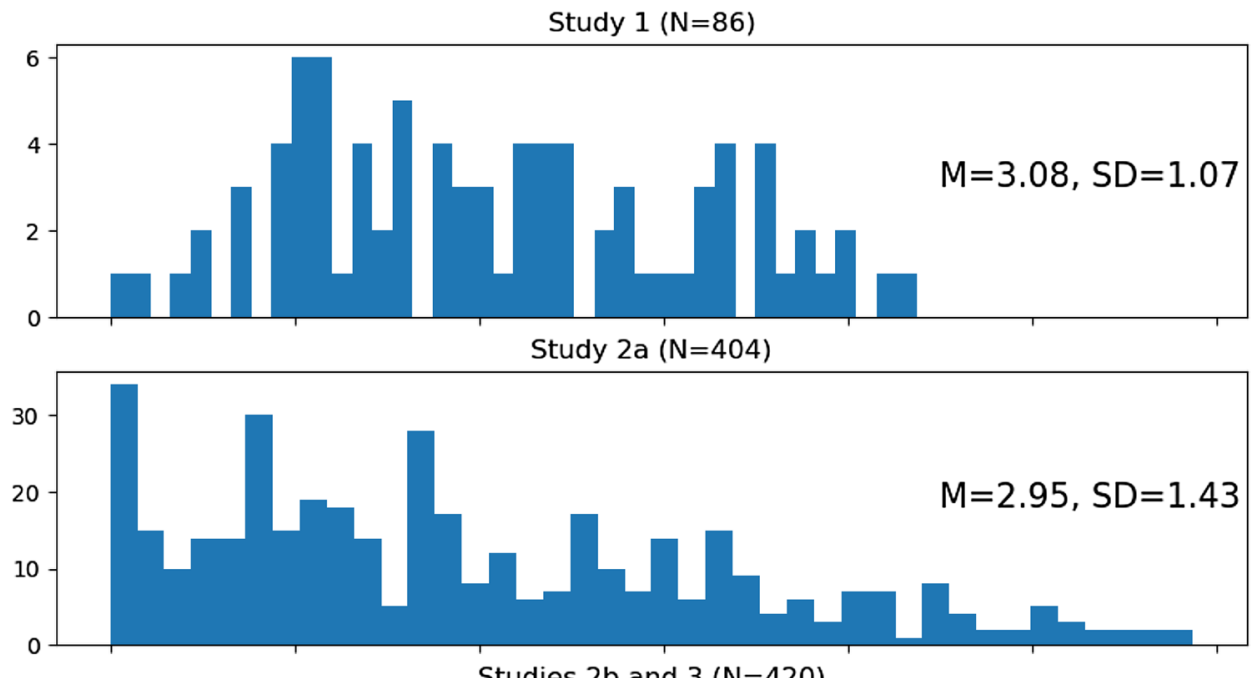

Studies $2 \mathrm{~b}$ and $3(\mathrm{~N}=420)$

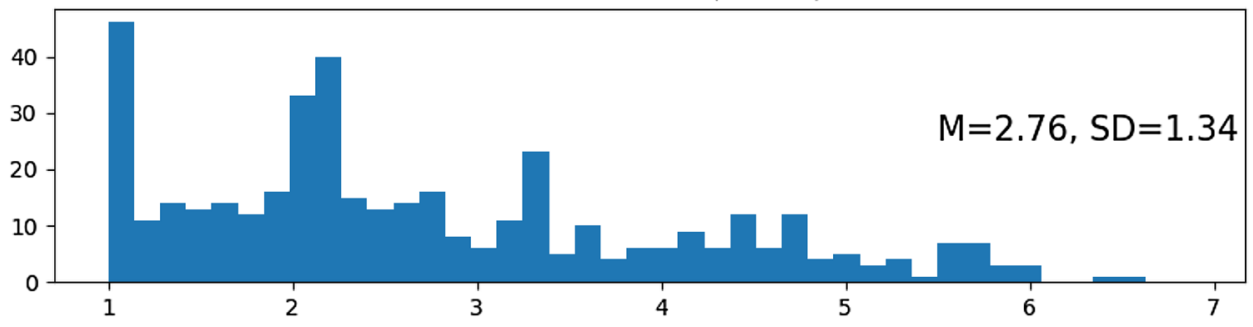

Funding This study was funded by NSERC Discovery grant.

Data availability Data for the studies are available at https://osf.io/ r4afz/.

\section{Declarations}

Conflict of interest The authors have no conflict of interest to declare.

\section{References}

Bechara A, Damasio AR, Damasio H, Anderson SW (1994) Insensitivity to future consequences following damage to human prefrontal cortex. Cognition. https://doi.org/10.1016/0010-0277(94)90018-3

Beitz KM, Salthouse TA, Davis HP (2014) Performance on the iowa gambling task: from 5 to 89 years of age. J ExpPsychol Gen 143(4):1677-1689. https://doi.org/10.1037/a0035823

Bench SW, Lench HC (2013) On the function of boredom. BehavSci. https://doi.org/10.3390/bs3030459

Bench SW, Lench HC (2019) Boredom as a seeking state: boredom prompts the pursuit of novel (Even Negative) experiences. Emotion 19(2):242-254. https://doi.org/10.1037/emo0000433

Biolcati R, Passini S, Mancini G (2016) "I cannot stand the boredom". Binge drinking expectancies in adolescence. Addictive Behaviors Reports 3:70-76. https://doi.org/10.1016/j.abrep.2016.05.001

Biolcati R, Mancini G, Trombini E (2018) Proneness to boredom and risk behaviors during adolescents' free time. Psychol Rep 121(2):303-323. https://doi.org/10.1177/0033294117724447
Blaszczynski A, McConaghy N, Frankova A (1990) Boredom proneness in pathological gambling. Psychol Rep 67(1):35-42. https:// doi.org/10.2466/pr0.1990.67.1.35

Boylan J, Seli P, Scholer AA, Danckert J (2020) Boredom in the COVID-19 pandemic: trait boredom proneness, the desire to act, and rule-breaking. Personality Individ Differ. https://doi.org/10. 1016/j.paid.2020.110387

Busemeyer JR, Stout JC (2002) A contribution of cognitive decision models to clinical assessment: decomposing performance on the Bechara gambling task. Psychol Assess. https://doi.org/10.1037// 1040-3590.14.3.253

Campbell JA, Samartgis JR, Crowe SF (2013) Impaired decision making on the balloon analogue risk task as a result of long-term alcohol use. J ClinExpNeuropsychol 35(10):1071-1081. https:// doi.org/10.1080/13803395.2013.856382

Carriere JSA, Cheyne JA, Smilek D (2008) Everyday attention lapses and memory failures: the affective consequences of mindlessness. Conscious Cogn. https://doi.org/10.1016/j.concog.2007.04.008

Dahlen ER, Martin RC, Ragan K, Kuhlman MM (2004) Boredom proneness in anger and aggression: effects of impulsiveness and sensation seeking. Personality Individ Differ 37(8):1615-1627. https://doi.org/10.1016/j.paid.2004.02.016

Dahlen ER, Martin RC, Ragan K, Kuhlman MM (2005) Driving anger, sensation seeking, impulsiveness, and boredom proneness in the prediction of unsafe driving. Accid Anal Prev 37(2):341-348. https://doi.org/10.1016/j.aap.2004.10.006

Danckert J (2019) Boredom: managing the delicate balance between exploration and exploitation. Boredom Your Mind. https://doi.org/ 10.1007/978-3-030-26395-9_3

Danckert J, Merrifield C (2018) Boredom, sustained attention and the default mode network. Exp Brain Res 236(9):2507-2518. https:// doi.org/10.1007/s00221-016-4617-5 
Danckert J, Mugon J, Struk AA, Eastwood J (2018) Boredom: what is it good for? In: Lench HC (ed) The function of emotions. Springer

Delorme A, Makeig S (2004) EEGLAB: an open sorce toolbox for analysis of single-trail EEG dynamics including independent component anlaysis. J Neurosci Methods 134:9-21. https://doi.org/10. 1016/j.jneumeth.2003.10.009

Eastwood JD, Frischen A, Fenske MJ, Smilek D (2012) The unengaged mind: defining boredom in terms of attention. PerspectPsycholSci 7(5):482-495. https://doi.org/10.1177/1745691612456044

Elhai JD, Vasquez JK, Lustgarten SD, Levine JC, Hall BJ (2018) Proneness to boredom mediates relationships between problematic smartphone use with depression and anxiety severity. SocSciComput Rev. https://doi.org/10.1177/0894439317741087

Farrell S, Lewandowsky S (2018) Computational modeling of cognition and behavior. Comput Model CognBehav. https://doi.org/10. 1017/CBO9781316272503

Fernie G, Cole JC, Goudie AJ, Field M (2010) Risk-taking but not response inhibition or delay discounting predict alcohol consumption in social drinkers. Drug Alcohol Depend. https://doi.org/10. 1016/j.drugalcdep.2010.05.011

Gardner W, Mulvey EP, Shaw EC (1995) Regression analyses of counts and rates: poisson, overdispersedpoisson, and negative binomial models. Psychol Bull. https://doi.org/10.1037/0033-2909.118.3. 392

Gehring WJ, Willoughby AR (2002) The medial frontal cortex and the rapid processing of monetary gains and losses. Science. https:// doi.org/10.1126/science.1066893

Gerritsen CJ, Toplak ME, Sciaraffa J, Eastwood J (2014) I can't get no satisfaction: potential causes of boredom. Conscious Cogn. https://doi.org/10.1016/j.concog.2013.10.001

Hanson KL, Thayer RE, Tapert SF (2014) Adolescent marijuana users have elevated risk-taking on the balloon analog risk task. J Psychopharmacol 28(11):1080-1087. https://doi.org/10.1177/ 0269881114550352

Hertwig R, Erev I (2009) The description-experience gap in risky choice. Trends CognSci. https://doi.org/10.1016/j.tics.2009.09. 004

Hooper CJ, Luciana M, Conklin HM, Yarger RS (2004) Adolescents' performance on the iowa gambling task: Implications for the development of decision making and ventromedial prefrontal cortex. Dev Psychol 40(6):1148-1158. https://doi.org/10.1037/ 0012-1649.40.6.1148

Hsu CF, Eastwood JD, Toplak ME, Liang JC, Hwang-Gu SL, Chen VCH (2020) Trait and state boredom: associations with attention failure in children with Attention-Deficit/Hyperactivity disorder. Psychiatry Res 286:112861. https://doi.org/10.1016/j. psychres.2020.112861

Humphries MA, Bruno R, Karpievitch Y, Wotherspoon S (2015) The expectancy valence model of the iowa gambling task: can it produce reliable estimates for individuals? J Math Psychol. https://doi.org/10.1016/j.jmp.2014.10.002

Hunter A, Eastwood JD (2018) Does state boredom cause failures of attention? Examining the relations between trait boredom, state boredom, and sustained attention. Exp Brain Res 236(9):24832492. https://doi.org/10.1007/s00221-016-4749-7

Isacescu J, Danckert J (2018) Exploring the relationship between boredom proneness and self-control in traumatic brain injury (TBI). Exp Brain Res 236(9):2493-2505. https://doi.org/10. 1007/s00221-016-4674-9

Isacescu J, Struk AA, Danckert J (2017) Cognitive and affective predictors of boredom proneness. CognEmot 31(8):1741-1748. https:// doi.org/10.1080/02699931.2016.1259995

Iso-Ahola SE, Crowley ED (1991) Adolescent substance abuse and leisure boredom. J Leis Res. https://doi.org/10.1080/00222216. 1991.11969857
Kahneman D, Tversky A (1979) Prospect theory: an analysis of decision under risk. Econometrica 47(2):263-292. https://doi.org/10. 2307/1914185

Kass SJ, Vodanovich SJ (1990) Boredom proneness: its relationship to type a behavior pattern and sensation seeking. Psychol J Hum Behav 12:10-23

Kılıç A, van Tilburg WAP, Igou ER (2020) Risk-taking increases under boredom. J BehavDecisMak 33(3):257-269. https://doi.org/10. 1002/bdm. 2160

Kurzban R, Duckworth A, Kable JW, Myers J (2013) An opportunity cost model of subjective effort and task performance. Behav Brain Sci 36(06):661-679. https://doi.org/10.1017/s0140525x12003196

Lejuez CW, Richards JB, Read JP, Kahler CW, Ramsey SE, Stuart GL, Brown RA (2002) Evaluation of a behavioral measure of risk taking: the balloon analogue risk task (BART). J ExpPsycholAppl 8(2):75-84. https://doi.org/10.1037/1076-898X.8.2.75

Lennon A, Oviedo-Trespalacios O, Matthews S (2017) Pedestrian selfreported use of smart phones: positive attitudes and high exposure influence intentions to cross the road while distracted. Accid Anal Prev. https://doi.org/10.1016/j.aap.2016.10.028

Lopez-Calderon J, Luck SJ (2014) ERPLAB: an open-source toolbox for the analysis of event-related potentials. Front Hum Neurosci 8:1-14. https://doi.org/10.3389/fnhum.2014.00213

Luce RD (1959) Individual choice behavior. John Wiley

MacPherson L, Reynolds EK, Daughters SB, Wang F, Cassidy J, Mayes LC, Lejuez CW (2010) Positive and negative reinforcement underlying risk behavior in early adolescents. PrevSci. https://doi.org/ 10.1007/s11121-010-0172-7

Malkovsky E, Merrifield C, Goldberg Y, Danckert J (2012) Exploring the relationship between boredom and sustained attention. Exp Brain Res 221(1):59-67. https://doi.org/10.1007/ s00221-012-3147-z

Martín R (2012) Event-related potential studies of outcome processing and feedback-guided learning. Front Hum Neurosci 6:1-40. https://doi.org/10.3389/fnhum.2012.00304

Matthies S, Philipsen A, Svaldi J (2012) Risky decision making in adults with ADHD. J BehavTherExp Psychiatry 43(3):938-946. https://doi.org/10.1016/j.jbtep.2012.02.002

Miao P, Li X, Xie X (2019) Hard to bear: state boredom increases financial risk taking. SocPsychol. https://doi.org/10.1027/18649335/a000408

Miltner WHR, Braun CH, Coles MGH (1997) Event-related brain potentials following incorrect feedback in a time-estimation task: evidence for a "generic" neural system for error detection. J CognNeurosci. https://doi.org/10.1162/jocn.1997.9.6.788

Miu AC, Heilman RM, Houser D (2008) Anxiety impairs decisionmaking: psychophysiological evidence from an Iowa Gambling Task. BiolPsychol 77(3):353-358. https://doi.org/10.1016/j.biops ycho.2007.11.010

Mugon J, Struk AA, Danckert J (2018) A failure to launch: Regulatory modes and boredom proneness. Front Psychol 9:1-7. https://doi. org/10.3389/fpsyg.2018.01126

Nelder JA, Mead R (1965) A Simplex Method for Function Minimization. Comput J. https://doi.org/10.1093/comjnl/7.4.308

Oxtoby J, Schroeter R, Johnson D, Kaye SA (2019) Using boredom proneness to predict young adults' mobile phone use in the car and risky driving. Transport Res Traffic PsycholBehav 65:457-468. https://doi.org/10.1016/j.trf.2019.08.008

Pfattheicher S, Lazarević LB, Westgate EC, Schindler S (2020) On the relation of boredom and sadistic aggression. J Personality Soc Psychol 52:120-145

Pleskac TJ, Wallsten TS, Wang P, Lejuez CW (2008) Development of an automatic response mode to improve the clinical utility of sequential risk-taking tasks. ExpClinPsychopharmacol 16(6):555564. https://doi.org/10.1037/a0014245 
Shurman B, Horan WP, Nuechterlein KH (2005) Schizophrenia patients demonstrate a distinctive pattern of decision-making impairment on the Iowa Gambling Task. Schizophr Res 72(2-3):215-224. https://doi.org/10.1016/j.schres.2004.03.020

Skues J, Williams B, Oldmeadow J, Wise L (2016) The effects of boredom, loneliness, and distress tolerance on problem internet use among university students. Int J Ment Heal Addict. https://doi. org/10.1007/s11469-015-9568-8

Struk AA, Scholer AA, Danckert J (2016) A self-regulatory approach to understanding boredom proneness. CognEmot 30(8):13881401. https://doi.org/10.1080/02699931.2015.1064363

Struk AA, Carriere JSA, Cheyne JA, Danckert J (2017) A short boredom proneness scale: development and psychometric properties. Assessment 24(3):346-359. https://doi.org/10.1177/1073191115 609996

Struk AA, Scholer AA, Danckert J, Seli P (2020) Rich environments, dull experiences: how environment can exacerbate the effect of constraint on the experience of boredom. CognEmot. https://doi. org/10.1080/02699931.2020.1763919

Tam KYY, van Tilburg WAP, Chan CS (2021) What is boredom proneness? A comparison of three characterizations. J Personal 15:1234. https://doi.org/10.1111/jopy. 12618

Tangney JP, Baumeister RF, Boone AL (2004) high self-control predicts good adjustment, less pathology, better grades, and interpersonal success. J Pers. https://doi.org/10.1111/j.0022-3506.2004. 00263.x

Wolff W, Martarelli CS (2020) Bored into depletion? Toward a tentative integration of perceived self-control exertion and boredom as guiding signals for goal-directed behavior. Perspect Psychol Sci. https://doi.org/10.1177/1745691620921394

Wolff W, Martarelli CS, Schüler J, Bieleke M (2020) High boredom proneness and low trait self-control impair adherence to social distancing guidelines during the COVID-19 pandemic. Int J
Environ Res Public Health 17(15):1-10. https://doi.org/10.3390/ ijerph17155420

Yakobi O, Cohen D, Naveh E, Erev I (2020) Reliance on small samples and the value of taxing reckless behaviors. Judgment Dec Making 10:24-43

Yakobi O, Boylan J, Danckert J (2021) Behavioral and electroencephalographic evidence for reduced attentional control and performance monitoring in boredom. Psychophysiology. https://doi.org/ 10.1111/psyp.13816

Yau YHC, Potenza MN, Mayes LC, Crowley MJ (2015) Blunted feedback processing during risk-taking in adolescents with features of problematic internet use. Add Behav 45:156-163. https://doi.org/ 10.1016/j.addbeh.2015.01.008

Yechiam E (2020) Robust consistency of choice switching in decisions from experience. JudgmDecisMak 15(1):74-81

Yechiam E, Zahavi G, Arditi E (2015) Loss restlessness and gain calmness: durable effects of losses and gains on choice switching. Psychon Bull Rev 22(4):1096-1103. https://doi.org/10.3758/ s13423-014-0749-4

Yeung N, Sanfey AG (2004) Independent coding of reward magnitude and valence in the human brain. J Neurosci. https://doi.org/10. 1523/JNEUROSCI.4537-03.2004

Zuckerman M, Eysenck SB, Eysenck HJ (1978) Sensation seeking in England and America: cross-cultural, age, and sex comparisons. J Consult ClinPsychol. https://doi.org/10.1037/0022-006X.46.1.139

Publisher's Note Springer Nature remains neutral with regard to jurisdictional claims in published maps and institutional affiliations. 